\title{
La Palma observations of umbral flashes
}

\author{
L. H. M. Rouppe van der Voort ${ }^{1}$, R. J. Rutten ${ }^{2,3}$, P. Sütterlin² ${ }^{2}$ P. J. Sloover ${ }^{2}$, and J. M. Krijger ${ }^{2}$ \\ 1 The Institute for Solar Physics of the Royal Swedish Academy of Sciences, AlbaNova University Center, \\ 10691 Stockholm, Sweden \\ 2 Sterrekundig Instituut, Utrecht University, Postbus 80 000, 3508 TA Utrecht, The Netherlands \\ e-mail: R.J.Rutten, P.Suetterlin,P.J.Sloover, J.M.Krijger@astro.uu.nl \\ 3 Institute of Theoretical Astrophysics, Oslo University, PO Box 1029 Blindern, 0315 Oslo, Norway
}

Received 23 December 2002 / Accepted 13 February 2003

\begin{abstract}
We present high-quality Ca II H \& K data showing chromospheric flashes in sunspot umbrae collected with the Swedish Vacuum Solar Telescope, the Dutch Open Telescope, and the Swedish 1-m Solar Telescope at the Roque de los Muchachos Observatory on La Palma. Differential movies, time slices, spectrograms, and Fourier power maps demonstrate that umbral flashes and running penumbral waves are closely related oscillatory phenomena, combining upward shock propagation with coherent wave spreading over the entire spot. We attribute the flash brightening to large redshift by post-shock material higher up. We find no obvious relation between umbral dots and umbral flashes.
\end{abstract}

Key words. Sun: sunspots - Sun: oscillations

\section{Introduction}

Umbral flashes are repetitive brightenings of the low umbral chromosphere in Ca II $\mathrm{H} \& \mathrm{~K}$ and other lines with roughly three-minute periodicity. Many observational and theoretical analyses followed their discovery by Beckers \& Tallant (1969). The extensive literature is summarised well in the introduction and various contributions in Thomas \& Weiss (1992), in particular the review by Lites (1992), and reviewed more recently by Staude (1999) and Bogdan (2000).

The recent literature exploits space observations, infrared spectropolarimetry, Stokes inversions, and numerical simulations to tie umbral flashes together with running penumbral waves, umbral plume oscillations, umbral fine structure, and wave conversion and reflection at magnetic canopies. We briefly list these developments.

The upward extension of chromospheric umbral oscillations in the form of higher-atmosphere oscillations above sunspots is intensively studied by the group at Oslo from ultraviolet data (e.g., Brynildsen et al. 2000, 2002; Maltby et al. 1999, 2001). Their results support the suggestion of Gurman et al. (1982) that upper-atmosphere three-minute modulation is not a resonance but a propagating-wave phenomenon, with these waves reaching the transition region in sunspot plumes above umbrae and even continuing in coronal loops sampled at EUV wavelengths by TRACE (cf. Schrijver et al. 1999) Confirmation comes from Nobeyama and VLA microwave

Send offprint requests to: L. Rouppe van der Voort, e-mail: rouppe@astro.su.se observations (Gelfreikh et al. 1999; Shibasaki 2001; Nindos et al. 2002).

The lateral extension of chromospheric umbral oscillations in the form of running penumbral waves is under scrutiny by Christopoulou et al. (1999, 2000, 2001) and Georgakilas et al. (2000, 2002). They diagnose both inward and outward running waves, and find from TRACE UV data that the outward running waves extend even into the moats surrounding sunspots.

The existence and nature of oscillations in the umbral magnetic field remain in debate (e.g., Lites et al. 1998). In a recent review, Staude (2002) considers rings of enhanced Fourier power in magnetic field strength at the umbra-penumbra boundary as well-established (e.g., Balthasar 1999; Kupke et al. 2000); they were compared to "whispering gallery" acoustics by Zhugzhda et al. (2000). However, Settele et al. (2002) warn against instrumental crosstalk. Infrared spectropolarimetry should be decisive; initial results indicate that opacity variations explain much of the small apparent field modulation (cf. Collados 2002).

Stokes inversion, a booming industry started by Ruiz Cobo \& Del Toro Iniesta (1992), was applied to umbral flash oscillations by Socas-Navarro et al. (2000a, 2000b, 2001). They find that a mixture of fast upward propagation and downward flow within the resolution element is needed to explain anomalous circular polarization, with the upward motions causing flashes. The linear polarization of umbral Ca II infrared lines requires a similar mixture, but also inhomogeneity or other ways to produce lateral wave spreading (López Ariste et al. 2001).

Forward simulation seems an intermediate step prior to data inversion that is needed to gain more understanding of the 
umbral dynamics underlying umbral flashes as well as running penumbral waves. In a recent review, Bogdan et al. (2002) take the Oslo simulations of a shaken fluxtube (Rosenthal et al. 2002) as a scale example of a complete sunspot to illustrate that it is naïve to try to identify sunspot oscillations as e.g., either magneto-acoustic or fast modes. Wave conversions and reflections cause a zoo of interference possibilities. There are no realistic sunspot oscillation simulations yet, but they are clearly needed to define and constrain likely wave modes and interference patterns.

Within this context of recent and expected progress exploiting new diagnostics and methods, this paper represents a rather traditional contribution. We present $\mathrm{Ca}$ II $\mathrm{H} \& \mathrm{~K}$ image and spectra sequences from various observing runs with various La Palma telescopes to furnish a classical inventory of umbral flash phenomenology. Familiar diagnostics produce familiar results; the various displays presented below generally confirm properties that have been noted already in the initial umbral flash and penumbral wave papers. However, our displays do so vividly and clearly thanks to high image quality, and they therefore merit presentation as an overview of chromospheric sunspot oscillation behaviour. The upcoming simulation efforts require such displays as proving ground to demonstrate their realism.

The most vivid, beautiful and fascinating visualisation of our data resides as movies at http: //www . edpsciences.org.

\section{Observations and reduction}

Observations. Our material consists of six sets of sunspot image sequences obtained with three solar telescopes on La Palma, respectively the former Swedish Vacuum Solar Telescope (SVST, Scharmer et al. 1985), its successor the Swedish 1-m Solar Telescope (SST, Scharmer et al. 2002), and the Dutch Open Telescope (DOT, Hammerschlag \& Bettonvil 1998). The SVST operation was terminated in August 2000. The DOT started observing in 1999. The SST became operational in March 2002, and was still stopped down to $60 \mathrm{~cm}$ aperture when these observations were taken. Table 1 specifies details for all six data sets. They are identified throughout this paper by the $A-F$ designations above the columns. In all displays solar North is up.

All six sets include image sequences obtained with narrowband interference filters centered on one of the Ca II H \& K lines at $396.9 \mathrm{~nm}$ and $393.3 \mathrm{~nm}$. They sample the low solar chromosphere. At both Swedish telescopes additional G-band (430.5 nm) images were taken simultaneously to provide a photospheric reference. The Ca II filtergrams from the SVST (sets $A, B$ and $C$ ) were obtained as slitjaw images for simultaneous $\mathrm{Ca}$ II $\mathrm{H}$ or $\mathrm{K}$ spectrometry with the Littrow spectrograph (Scharmer et al. 1985). The latter was used with a $25 \mu \mathrm{m}(0$.'23) wide slit, yielding slit-limited spectral resolution corresponding to a $3 \mathrm{pm}$ passband. Spectrograms containing the Ca II $\mathrm{K}$ core were recorded with data sets $A$ and $B$, spectrograms containing $\mathrm{Ca}$ II $\mathrm{H}$ with data set $C$. The spectrogram pixels measured $1.1 \mathrm{pm}$ in the spectral and 0 !'$^{\prime} 083$ in the spatial direction. Precise correspondence between the spectrograms and the
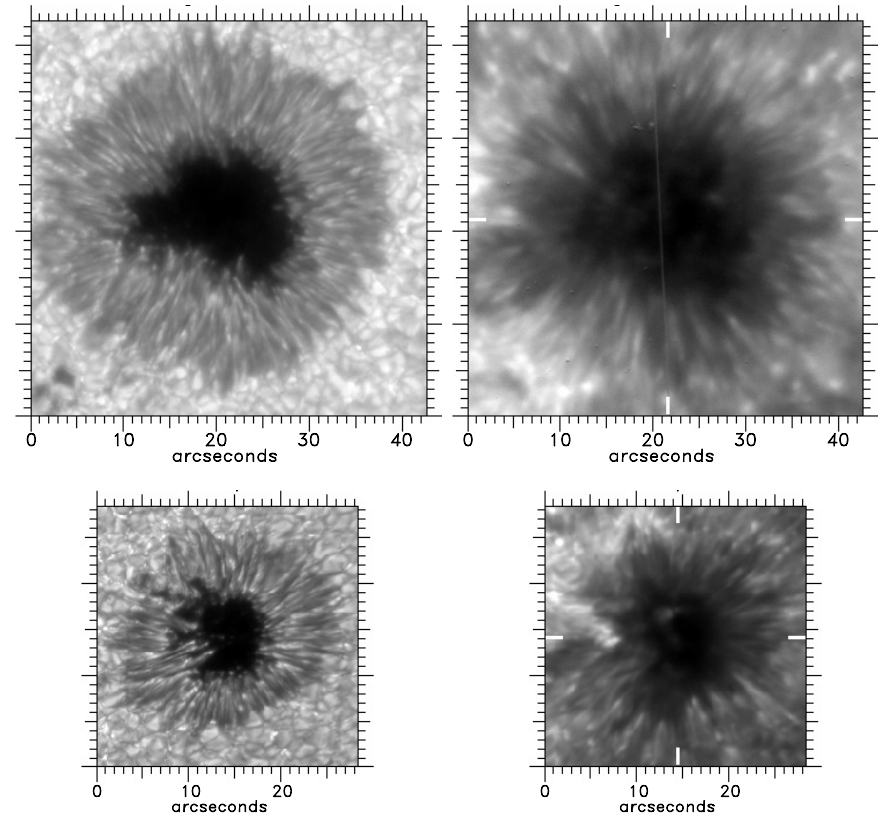

Fig. 1. Sample images from data sets $C$ (upper panels) and $F$ (lower panels). Lefthand panels: G-band filtergrams. Righthand panels: simultaneous Ca II H filtergrams. The white markers specify cut locations for Fig. 3.

simultaneous Ca II K slitjaw images was obtained by using synchronized CCD cameras, but there was slight differential position variation due to spectrograph instability. More details on the reduction of the spectrograph data are given in Rouppe van der Voort (2002). The presence of the spectrograph slit can be discerned in the images and in some of the resulting Fourier displays below. The other data sets have better image quality.

The imaging with the two Swedish telescopes employed frame selection (Scharmer \& Löfdahl 1991) in which the best image (i.e., with the highest contrast) was selected from a continuous stream of images during a preset interval. The latter varied between 8 and $20 \mathrm{~s}$ between the different Swedish data sets. The precise timing within the intervals has random character.

The DOT employed speckle data acquisition with subsequent image reconstruction using the speckle masking technique of Weigelt (1977), Hofmann \& Weigelt (1986), and von der Lühe (1984). Speckle bursts of 80 frames each were taken at 8 frames $\mathrm{s}^{-1}$ rate by a fast CCD-camera and processed off-line using the Göttingen speckle reconstruction code (e.g., de Boer \& Kneer 1992; de Boer 1993, 1996; Sütterlin \& Wiehr 2000), yielding one reconstructed image per burst which is corrected for the seeing and the DOT pupil mask. Their quality approaches the diffraction limit of the telescope when the seeing is reasonably good.

Figure 1 shows sample images from data sets $C$ and $F$, respectively from the SVST and SST. The Ca II H images are less sharp then the G-band images, partially due to longer exposure but also from scattering in the $\mathrm{H}$ line and possibly from intrinsically coarser fine structure. 
Table 1. Observation details. Sunspot group classification according to McIntosh (1990), magnetic type in the Mount Wilson classification.

\begin{tabular}{lllllll}
\hline \hline data set & $A$ & $B$ & $C$ & $D$ & $E$ & $F$ \\
\hline date & 20 Sep. 1999 & 20 Sep. 1999 & 9 Aug. 2000 & 1 Nov. 2000 & 2 Nov. 2000 & 30 Apr. 2002 \\
NOAA AR number & 8704 & 8704 & 9115 & 9214 & 9214 & 9919 \\
group classification & Cso & Cso & Cao & Eao & Eao & Cso \\
magnetic type & $\beta$ & $\beta$ & $\beta$ & $\beta$ & $\beta$ & $\beta$ \\
location [ ${ }^{\circ}$ ] & S22 E37 & S22 E32 & N18 E14 & S11 E20 & S11 E07 & N15 W13 \\
observing angle $\theta\left[^{\circ}\right.$ ] $(\mu)$ & $40(0.77)$ & $40(0.77)$ & 14 (0.97) & $23(0.92)$ & $14(0.97)$ & $31(0.86)$ \\
telescope & SVST & SVST & SVST & DOT & DOT & SST \\
start time [UT] & $10: 45: 13$ & $16: 02: 34$ & $8: 22: 13$ & $10: 34: 07$ & $08: 58: 00$ & $17: 32: 52$ \\
duration [hms] & $01: 02: 15$ & $01: 02: 07$ & $01: 09: 51$ & $00: 22: 07$ & $00: 31: 20$ & $00: 47: 48$ \\
cadence [s] & 8.47 & 8.98 & 17.6 & 15.6 & 15.7 & 23.7 \\
Ca filter (width [nm]) & Ca II K $(0.3)$ & Ca II K $(0.3)$ & Ca II H $(0.1)$ & Ca II H (0.1) & Ca II H $(0.1)$ & Ca II H (0.1) \\
pixelsize [" $]$ & 0.063 & 0.063 & 0.063 & 0.163 & 0.0815 & 0.0663 \\
exposure time: Ca [ms] & 150 & 150 & 750 & 8 & 8 & 181 \\
G-band filter: width [nm] & 1.2 & 1.2 & 1.2 & - & - & 1.1 \\
exposure time: G-band [ms] & 40 & 40 & 12 & - & - & 4 \\
spectrometry & Ca II K & Ca II K & Ca II H & - & - & - \\
\hline
\end{tabular}

Reduction. Flat-field correction and subtraction of bias and dark current were applied to all images. All images per sequence were aligned to a reference one to correct for telescope jitter, pointing errors, and seeing-induced image shifts. For the Swedish telescopes, which have alt-azimuth turrets, the alignment procedure also included removal of the diurnal field rotation. This is not necessary for DOT sequences since this telescope has a parallactic mount.

The smaller-scale geometric deformations that are introduced by atmospheric seeing as time-varying refractive differences between different isoplanatic patches across the field of view were corrected by destretching following Shine et al. (1994). This technique tesselates each image into a grid of overlapping subimages (square subfields of about $8^{\prime \prime}$ width) which are sequentially cross-correlated, each subfield to the corresponding subfield in the previous image, to determine sequences of spatial offset vectors per subfield. The offset signals contain contributions from seeing and from intrinsic horizontal motions over the solar surface. The subfield jitter caused by seeing has high-frequency signature and is isolated by subtracting a five-minute running mean from each temporal offset sequence. Linear interpolation between the subfield sequences delivers the jitter excursions for each pixel per time step. Bicubic spatial interpolation is then applied to correct each image for these motions.

We found that this destretching produces overcorrections in the umbral parts of our Ca II H \& K filtergrams due to the presence of the high-contrast umbral flashes and oscillations. The brightness waves move so fast in lateral directions that the resulting high-frequency subimage offsets can not be separated cleanly from the seeing shifts. In our initial power maps we noted striking rings of enhanced Fourier power at the inner penumbral boundary, rather like the boundary rings of power in magnetic field strength in Fig. 4 of Balthasar (1999; reprinted in Staude 2002). However, in our case these apparent power rings turned out to be artifacts caused by the combination of steep gradients and fast intrinsic motions with our destretching technique. They went away when the destretching was turned off.

We therefore refrained from destretching the umbral areas in our $\mathrm{H} \& \mathrm{~K}$ filtergram sequences. However, it should be noted that at the Swedish telescopes a correlation tracker (Shand et al. 1995) was employed using the umbra itself as target, which significantly reduced the full-image jitter as well as the subfield jitter near the target area. The residual subfield offsets are estimated to have rms values below 3 px $\sim 0$ ". 2 in the umbra. For the DOT image sequences the speckle reconstruction suppresses differential image motion to residual rms subimage offsets below 0 ". 1 .

\section{Results}

Movies. The most direct way to appreciate the umbral flash phenomenon in $\mathrm{H} \& \mathrm{~K}$ image sequences is to view movies constructed from such images, in particular after subtraction of a running-mean image, i.e., the temporal average per pixel over the whole sequence or over some appropriate shorter time span, for example five minutes (Alissandrakis et al. 1992; Brisken $\&$ Zirin 1997). We provide such mean-subtracted Ca II movies as mpeg files at http: //www . edpsciences.org and urge the reader to inspect these (in particular the movies from sets $D-F$ and preferably with a frame-steppable movie player). They are beautiful in their own right, and they illustrate many aspects of umbral flashes and running penumbral waves described in the sunspot literature with unusual clarity, thanks to superior image quality and registration.

The movies vividly demonstrate the dynamic nature of the umbral three-minute oscillation. In all six movies, bright umbral flashes continuously appear as rather localised intensity enhancements at varying locations within the umbra. The flashes seem always superimposed on an already pre-existing and rapidly changing brightness pattern consisting of strands of brightness "dancing around" the umbra. Most bright flashes are 

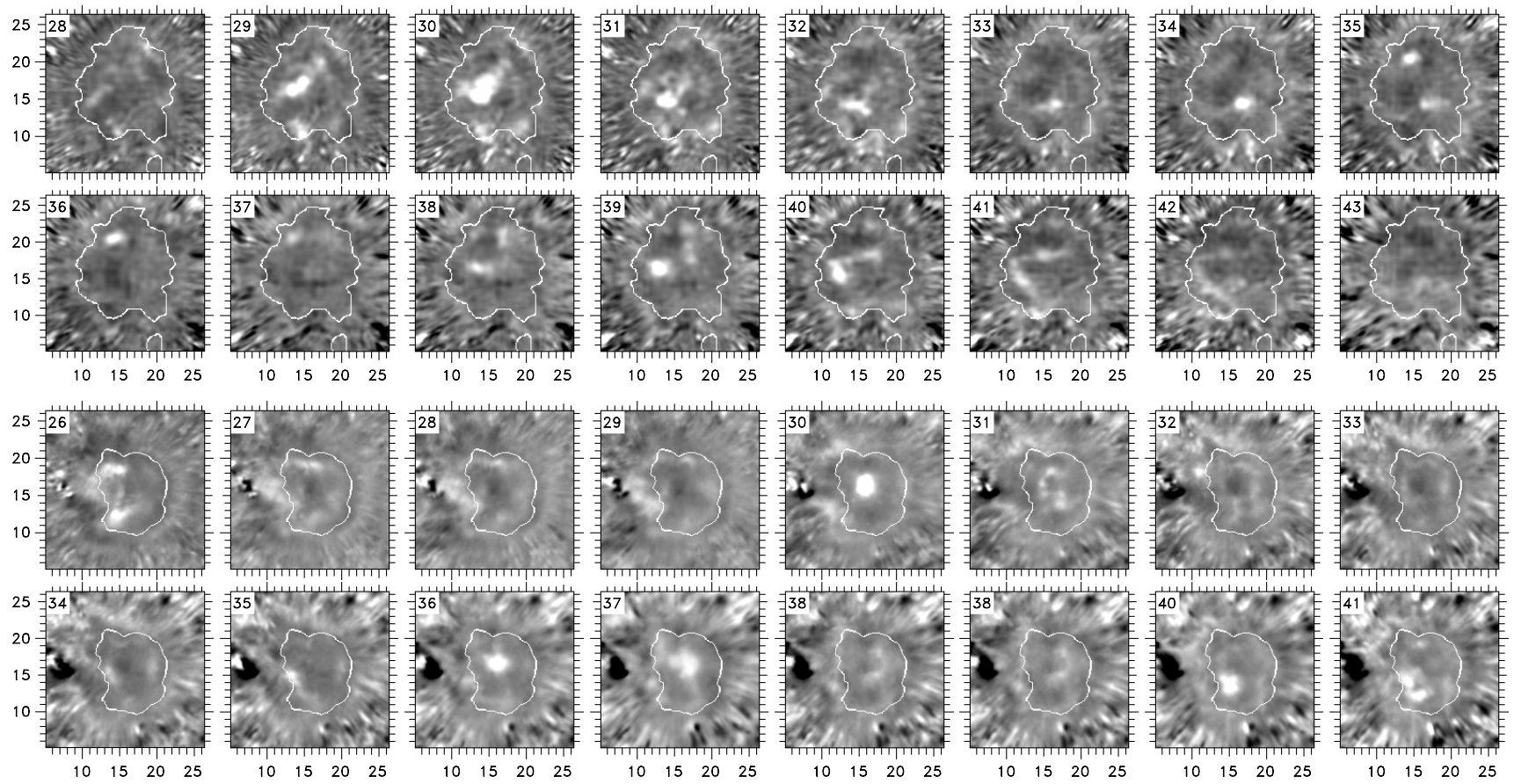

Fig. 2. Two Ca II H cut-out sequences from data sets $D$ (DOT) and $F$ (SST). A five-minute running mean is subtracted from each image. The cadence is $15.8 \mathrm{~s}$ between successive frames for the first sequence, irregular with a mean interval of $23.7 \mathrm{~s}$ for the second sequence. Axes: spatial scales in arcsec. The contours mark the umbral boundary. The greyscale is clipped so that the flashes are overexposed. Complete movies at http://www. edpsciences.org.

followed in our mean-subtracted movies by exceptionally dark patches covering the flash location, but that is more a result of the bright flash enhancing the 5-min running mean than of the post-flash minimum being particularly low. Around the dark patches one sees rapidly expanding arcs of brightness, sometimes nearly circular and concentric with the umbra for a central flash, but more often outlining partial arcs with smaller radius, usually on the penumbral side. Flashes can go off in pairs, multiple arcs may be spreading simultaneously; often, possibly always, the umbral brightness pattern is made up of constructively interfering expanding post-flash rings and arcs. They can sometimes be followed through the penumbra to near its outer edge. Not all flashes produce expansion arcs that travel that far, but by and large there is no real difference between the postflash expansion arcs within the umbra and in the penumbra. Thus, running penumbral waves and umbral flashes are manifestations of the same oscillatory phenomenon.

Figure 2 shows cutout sequences selected from two differential CaII $\mathrm{H}$ movies. The first panel of the upper sequence shows a pre-flash umbra with a characteristic patchy $\mathrm{Ca}$ II $\mathrm{H}$ brightness distribution. A double flash goes off in the next panel (frame 29 in the movie) and then expands into a larger one. In frame 31 the blob of emission appears to shift downward while two dark patches have appeared precisely at the original double-flash locations, each surrounded by a small bright ring. In frame 33 the dark patch has grown to be the reversal of the bright patch in frame 30 . The small dark area to its lower right in frame 30 evolves in counter phase to a flash in frame 34 , joined by a similar counter-phase flash in frame 35 . In the meantime the bright rings from the double flash have spread out to well into the penumbra, as seen much better on the movie. The brightness pattern in frame 38 resembles the initial one in frame 28. A new flash develops near the site of the double one. Frame 42 contains multiple spreading brightness rings within the umbra.

The second sequence shows a bright central flash in frame 30 surrounded by an irregular ring of emission from a preceding flash (frames 23-24, not shown) that has spread out and reached the penumbra in the meantime. The frame 30 flash spreads a new ring; another flash appears in the same location at frame 36. By frame 41 its spreading ring is well into the penumbra.

The systematic pattern of a bright flash followed by a co-local dark patch and spreading brightness arcs vividly reminds one of the splash from dropping a stone in a pond and strongly suggests upward propagation of a disturbance along funnel-shaped field lines, with the vertically upward propagating part the first to reach nonlinear amplification height within the $\mathrm{H} \& \mathrm{~K}$ response layer and the more slanted parts of the disturbance front getting there later and not as high. We suspect that the extreme brightness of the flash is caused by radiative transfer enhancement when the line of sight is along the propagation direction (see below).

Time slices. Figure 3 shows Ca II $\mathrm{H}$ brightness modulation in planar $\left(x_{0}, y, t\right)$ and $\left(x, y_{0}, t\right)$ cuts through the differential movies $C-F$, i.e., the temporal evolution of the mean-subtracted $\mathrm{H} \& \mathrm{~K}$ brightness for all pixels along linear cuts in the $x$ or $y$ direction. They illustrate clearly that umbral flashes are always enhancements of a larger-scale three-minute brightness oscillation of which the modulation patterns in these space-time 

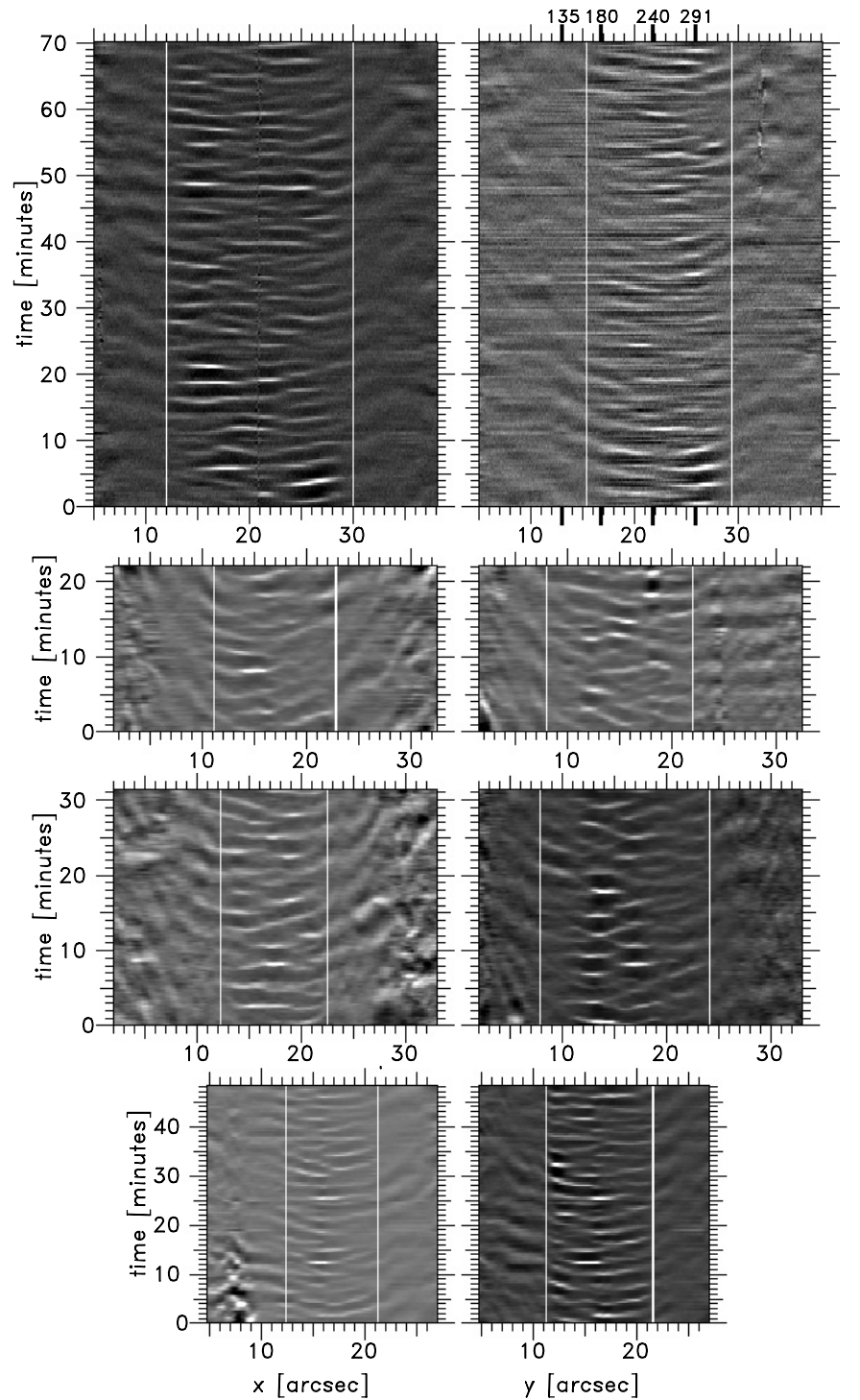

Fig. 3. Spatio-temporal "time slice" diagrams from data sets $C, D$, $E$ and $F$ (from top to bottom) showing $\mathrm{Ca}$ II $\mathrm{H}$ brightness modulation along image cuts in the $x$ direction (lefthand panels) and $y$ direction (righthand panels). For $D$ and $F$ the cut locations are specified in Fig. 1. A five-minute running mean was subtracted for each pixel. The vertical lines mark the umbra boundary. The top righthand panel shows horizontal interference from the presence of the spectrograph slit at this $x$ position. The numbered markers above the panel specify slit sample locations for Fig. 4.

diagrams assume bowl shapes that usually extend across the whole umbra and often over the penumbra as well. The uptilted bowl walls correspond to the bright-arc spreading observed in the movies, sampling the spread motion along the cut row or column. Concentric rings produce stacked bowls with steeper walls for slower outward propagation. The umbral slopes indicate spread velocities of $10-15 \mathrm{~km} \mathrm{~s}^{-1}$, sometimes well above $20 \mathrm{~km} \mathrm{~s}^{-1}$. The running penumbral waves propagate outward at 4-7 $\mathrm{km} \mathrm{s}^{-1}$, occasionally faster than $10 \mathrm{~km} \mathrm{~s}^{-1}$.

The Ca II movies give a strong visual impression that flashes act as triggers to the subsequent wave spreading. Indeed, the running waves are often described in the literature as caused by umbral flashes. However, the time slices in Fig. 3 demonstrate that flashes are actually part of the running wave phenomenon. They often occur in the more horizontal parts of the time-slice ridges, and then appear stationary during their brief lifetime, but they may also appear as enhancements of the tilted ridge parts and exhibit fast proper motion. Invariably the flashes are part of the ridges, and must therefore be seen as local enhancements of the umbral three-minute oscillation. They tend to reappear at the same place a number of times in short wave trains, but seem to occur rather randomly in the umbra over longer time scales.

A more quantitative analysis of similar patterns in Ca II $854.2 \mathrm{~nm}$ time slices is given by Tziotziou et al. (2002).

Dopplershift charts. Figure 4 is produced from Ca II $\mathrm{H}$ spectrograms taken as part of data set $C$. The central emission feature displayed by the $\mathrm{Ca}$ II $\mathrm{H}$ line in umbrae sweeps back and forth in wavelength with time in non-sinusoidal $Z$ patterns. This sawtooth behaviour was depicted graphically already by Beckers \& Tallant (1969) and Kneer et al. (1981), and displayed in greyscale Doppler-chart format and called Z pattern by Thomas et al. (1984).

The line core excursions during the three-minute oscillations reach peak-to-peak amplitudes up to $15 \mathrm{~km} \mathrm{~s}^{-1}$, considerably larger than the $6 \mathrm{~km} \mathrm{~s}^{-1}$ value mentioned by Lites (1992). The flashes occur toward the end of the rapid (near-horizontal) returns from maximum redshift to maximum blueshift and are followed by gradual motion back to maximum redshift. The pattern is sharpest in the center area of the umbra (pixel 240). The other charts show instances of interference between multiple waves.

The $Z$ pattern was attributed to shock waves when noted at larger amplitude $\left(10 \mathrm{~km} \mathrm{~s}^{-1}\right)$ in comparable He I $1083 \mathrm{~nm}$ spectrometry by Lites (1986, cf. Fig. 3 of Collados 2002) and was compared to the Oslo simulations of internetwork oscillations already by Lites (1994). The diagnostic internetwork Ca II H line-formation diagrams through which Carlsson \& Stein $(1994,1997)$ analysed their simulations indeed suggest that the umbral Z-patterns and flashes come from analogous shock behaviour, with the fast blueward excursions describing upward propagating shocks and the slower redward excursions post-shock fall-back. The flash intensity enhancement during the blueshift is likely to be set nonlinearly and to involve line-center opacity removal along the line of sight by even larger redshifts in overlying layers, caused by material that falls back after preceding shocks have passed through on their way up - the same mechanism through which preceding shocks are part of nonlinear internetwork $\mathrm{K}_{2 V}$ grain brightening (cf. Rutten 1996). This implies that the occurrence of flashes along the time-slice ridges in Fig. 3 is likely to reflect the presence of large Dopplershifts higher up rather than exceptional local wave heating, and that alignment of the line of sight with the wave propagation direction produces maximum visibility.

The Dopplershift chart in the rightmost panel in Fig. 4 is for a location in the penumbra just outside the umbral boundary, as indicated in the sample image at left. The central peak reaches less contrast relative to the inner line wings. The $Z$ pattern is 

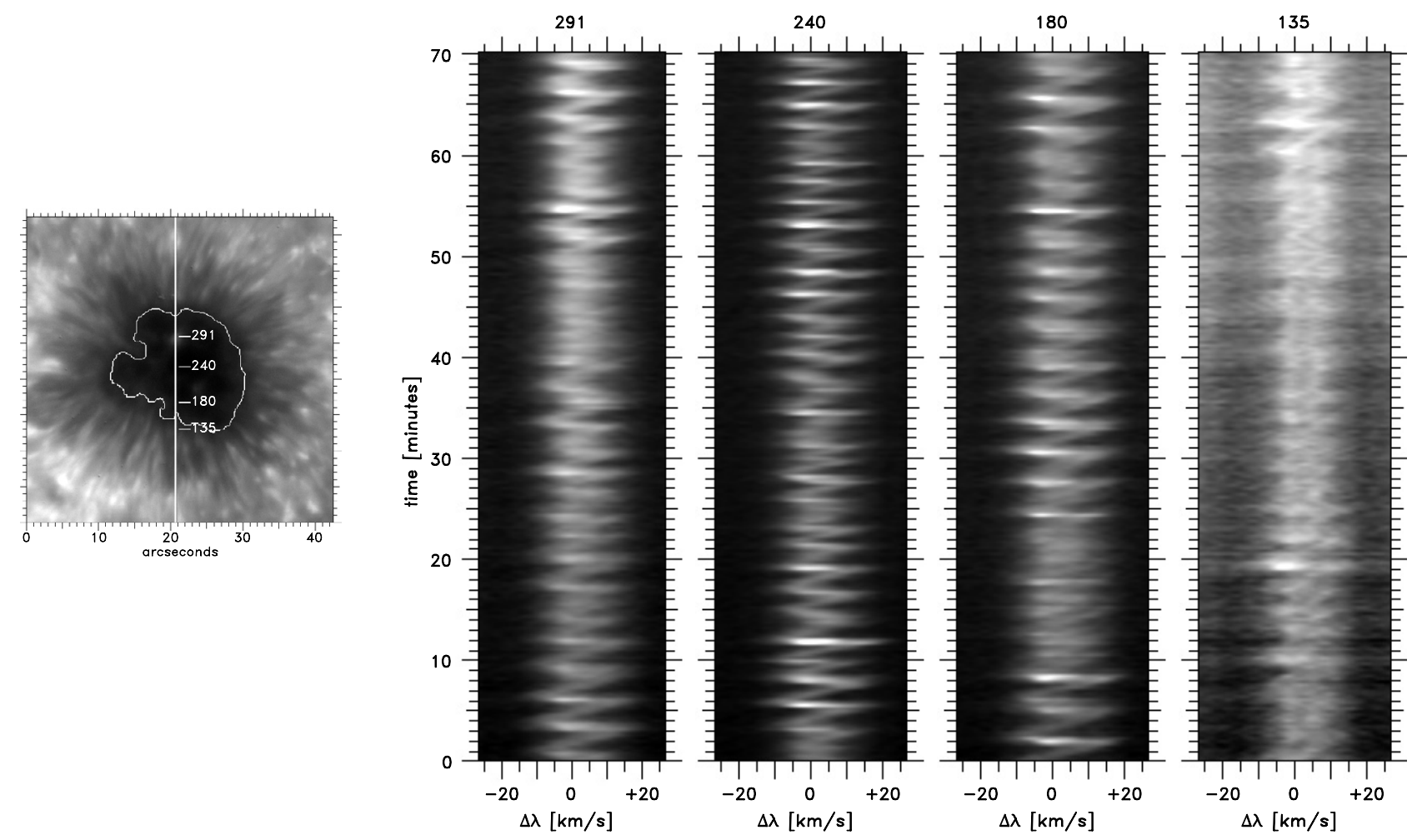

Fig. 4. Ca II H Dopplershift charts from data set $C$. The Ca II H image (left) specifies four slit sample locations, with pixel numbers. They correspond to the four marked columns in the top right panel of Fig. 3. The four strip charts show the temporal evolution of a short spectral segment containing the central emission feature of the $\mathrm{Ca}$ II $\mathrm{H}$ line at these locations. The fourth chart samples the inner penumbra close to the umbral boundary.

less sharp but still present, implying that also running penumbral waves possess similar shock-like behaviour along the line of sight.

Each flash in the Dopplershifts charts can be found back along the corresponding sampling column in the top-right time slice in Fig. 3 (with some brightness differences due to slit position variations caused by spectrograph instability). For example, the bright flash near $t=8 \mathrm{~min}$ in the third chart (pixel 180) is part of a curved brightness ridge in Fig. 3 which has flashes on both sides of the umbra and continues well into the penumbra, also on both sides. The Dopplershift charts show a corresponding $\mathbf{Z}$ pattern at each sample location, also the penumbral one (pixel 135, $t=10 \mathrm{~min}$ ). Thus, the waves retain Dopplershift shock signature while they spread out toward and into the penumbra.

Power maps. Figure 5 shows Fourier power maps for each Ca II image sequence. In each group of four pairs of diagrams, the first pair shows the time-averaged $\mathrm{H}$ or $\mathrm{K}$ intensity, the second pair the power distribution for 5-min modulation, the third pair for 3-min modulation, the final pair for 2-min modulation. Each pair consists of a map, with logarithmic greyscaling for the power maps, and a tracing of the power along the indicated horizontal cut on a linear scale normalized by the spatial average. The umbral contours defined by the averagedintensity maps are overlaid on the power maps and also added to Figs. 2-4 for reference.
The five-minute power maps show umbrae dark, the shorter-period maps have umbrae power-bright. In all cases the spatial distributions are rather patchy. The three-minute and two-minute maps and tracings show roughly similar patterns. Together, they describe the spatial occurrence distribution of the umbral flashes. The linear-scale tracings above the power maps show that this distribution is often markedly peaked: umbral flash power tends to be fairly concentrated, often being larger in the umbral interior than near the penumbra. However, the peaks are wide enough and show sufficient morphological variation between the different data sets that one cannot conclude that umbral flashes have mono-location pistons. There is no indication of increased brightness power at the umbrapenumbra boundary (as noted above, we found quite striking power rings initially but regard these as artifacts).

Umbral flashes versus umbral dots. The non-homogeneous power distributions in Fig. 5 suggest a search for flash localising agents in the underlying photosphere. This can be done for the four Swedish-telescope data sets which contain simultaneous G-band image sequences. In particular, we were curious to test whether there is spatial coincidence or avoidance between umbral dots and flashes. For example, Spruit (1981) has suggested that flashes are overstable oscillations from convective overshoot in field-free columns marked by umbral dots as in the Parker (1979) "octopus" model for sunspot structure.

Figure 6 shows results in the form of time-averaged G-band and $\mathrm{Ca}$ II $\mathrm{H}$ or $\mathrm{K}$ brightness maps that are clipped in brightness 

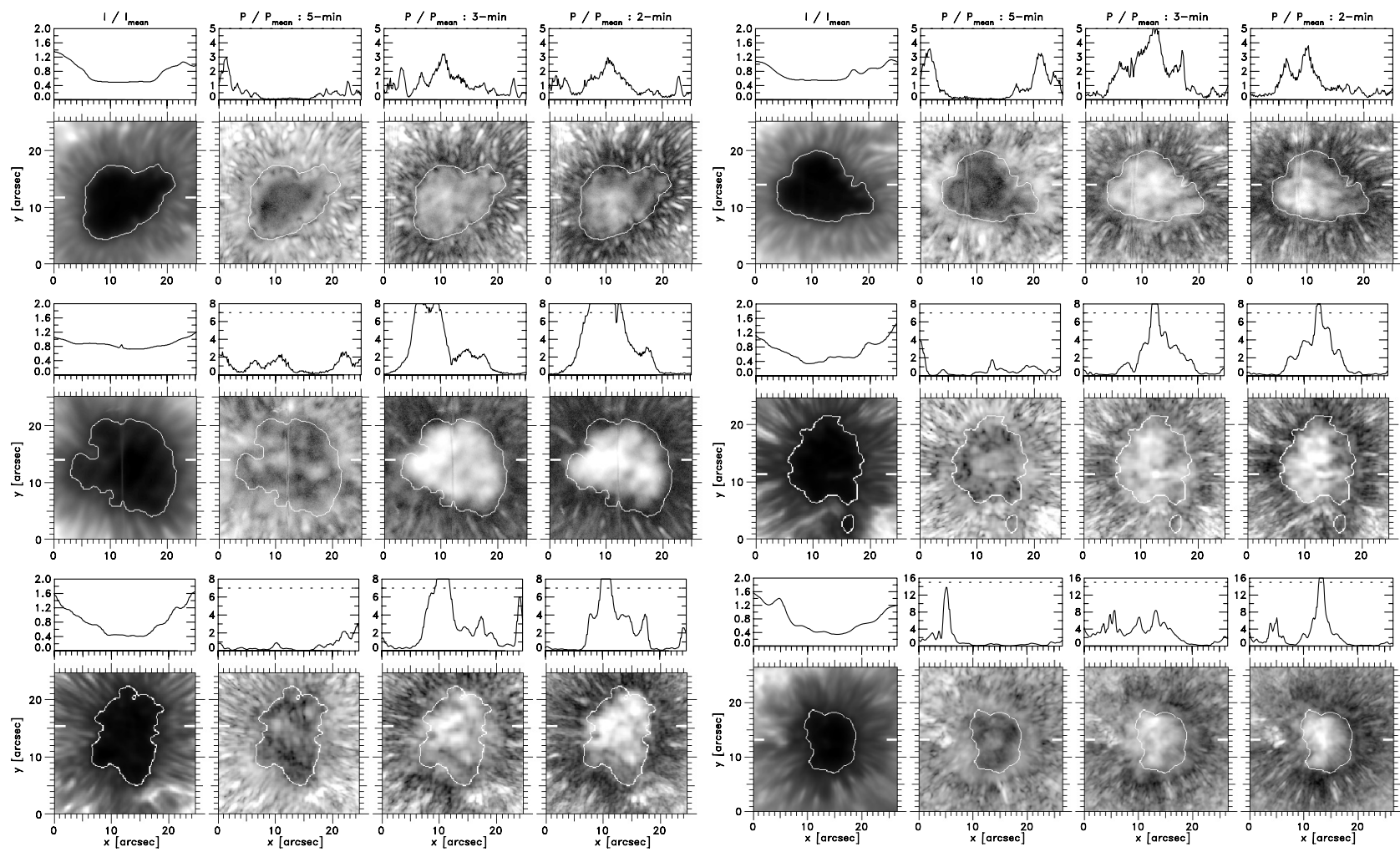

Fig. 5. Fourier power maps from data sets $A$ and $B$ (upper row), $C$ and $D$ (middle row), and $E$ and $F$ (bottom row). For each set the first column shows time-averaged intensity per pixel normalized by its spatial mean over the selected subfield, the second column shows five-minute power per pixel normalized by its spatial mean, the third and fourth columns show the same for three-minute and two-minute power. The greyscale of the power maps is logarithmic in order to bridge large contrasts. The profiles show the intensity or power along the horizontal cuts indicated by white markers, on linear scales and with greyscale cutoffs indicated by dashed lines. The contours mark the umbral edge as reference.
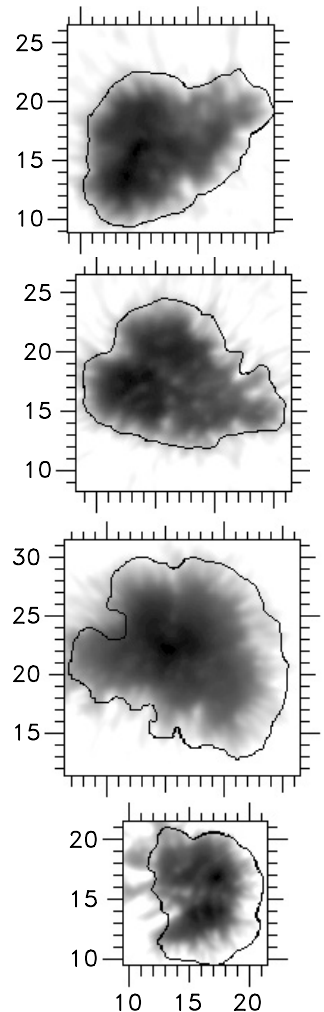
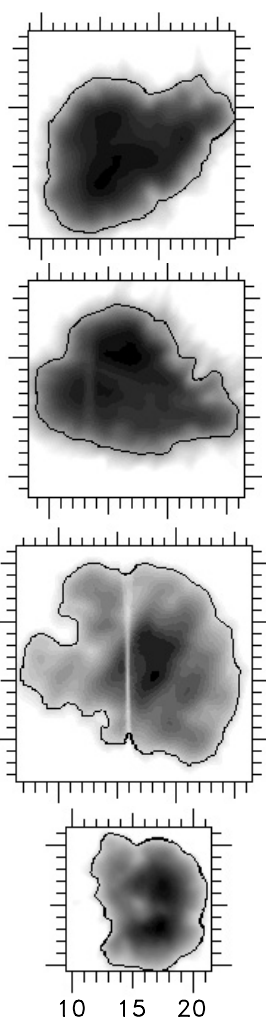
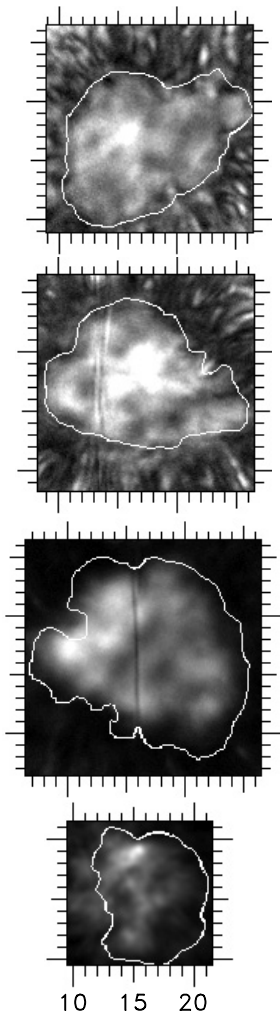
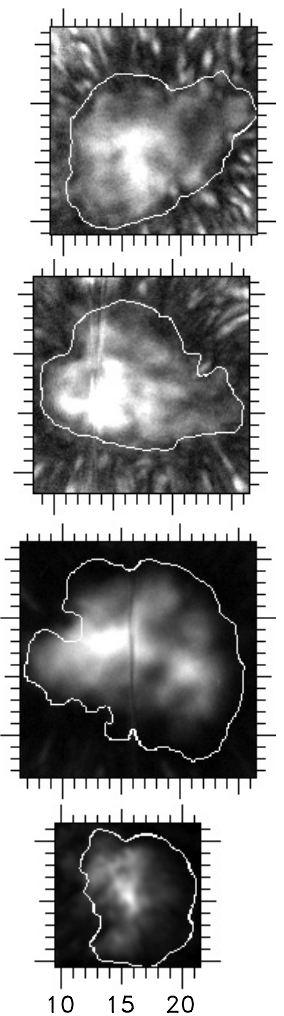

Fig. 6. Search for a relationship between photospheric and chromospheric umbral fine structure from data sets $A, B, C$ and $F$. The first column shows the time-averaged G-band brightness, the second column the time-averaged $\mathrm{Ca}$ II $\mathrm{H}$ or $\mathrm{K}$ brightness, the third and fourth column show the same three-minute and two-minute power maps as in Fig. 5 but with linear greyscaling. Axes: spatial scales in arcsec. The vertical stripes are due to the spectrograph slit in the Ca II slitjaw images. 
scale to emphasize the umbrae. The other panels are two- and three-minute $\mathrm{H}$ or $\mathrm{K}$ brightness power maps as in Fig. 5, but now with linear greyscale. Comparison indicates that umbral flashes seem to favour the dark umbral interior in the upper two sets, but to the contrary avoid the dark parts in the lower two sets. The test remains inconclusive.

\section{Discussion}

The principal result stemming from our La Palma image sequences is the close linkage between umbral flashes and running penumbral waves at the upper-photosphere/lowchromosphere heights sampled in Ca II H\& K filtergrams. Observed in this diagnostic, umbral flashes and running penumbral waves belong to a common and ubiquitous oscillatory phenomenon with wave fronts spreading in arcs over the umbra and penumbra. The fronts apparently spread out horizontally but are likely to actually propagate upwards along the divergent funnels and filamentary channels defined by the sunspot fields (as indeed indicated by $\mathrm{H} \alpha$ Doppler asymmetry, e.g., Christopoulou et al. 1999).

The flashes are local enhancements of the oscillation pattern. When appearing stationary and at the center of the spreading rings of $\mathrm{H} \& \mathrm{~K}$ emission, flash intensities reach particularly high values, indicating that maximum brightness modulation amplitude is reached through vertical propagation - as holds for the oscillations observed higher up in upright umbral plumes and umbra-connected coronal loops by e.g., Schrijver et al. (1999), Maltby et al. (2001) and Brynildsen et al. (2002). As noted above, we suspect that the brightness enhancements in the flashes stem from upward propagation along the line of sight through Dopplershift removal of line-center opacity in higher-up back-falling matter left after the passage of preceding shocks, comparably to internetwork $\mathrm{K}_{2 V}$ grain formation as proposed first by Athay (1970) and diagnosed in detail by Carlsson \& Stein (1997).

Our various displays therefore seem to confirm the frequently made suggestion that umbral flashes represent nearacoustic field-guided upward-propagating shock waves. The fast-spreading umbral rings and slower-spreading running penumbral waves may mark later wave front arrival at the $\mathrm{H} \& \mathrm{~K}$ sampling height due to longer travel path along divergent sunspot field lines.

At the same time, our movies illustrate the complexity that must necessarily go into numerical simulations in order to reproduce and interpret these sunspot oscillations. Onedimensional radiation-hydrodynamic acoustic wave simulation sufficed to reproduce the internetwork oscillations observed by Lites et al. (1993) in the numerical modelling of Carlsson \& Stein (1997), with magnetic-canopy complexity only appearing with the advent of higher-up diagnostics and multi-dimensional simulation (McIntosh et al. 2001; McIntosh \& Judge 2001; Rosenthal et al. 2002). As pointed out forcefully by Bogdan et al. (2002), sunspot wave simulation necessarily needs to include magnetism, multi-dimensional field geometry, canopy effects, and systematic flows; intricate wave interference and mode conversion may play key roles. Nevertheless, wave behaviour as depicted in our movies should lend itself to such numerical reproduction and so become interpretable in detail.

We suspect that, just as in the case of the internetwork three-minute oscillation, the hardest nut to crack may be to identify the piston agents. Figure 6 suggests that umbral dots do not play an obvious role.

Acknowledgements. The former SVST, the DOT and the SST are part of the Spanish Observatorio del Roque de los Muchachos of the Instituto de Astrofísica de Canarias. The SVST/SST is operated by the Royal Swedish Academy of Sciences. The DOT is funded by Utrecht University, The Netherlands Organisation for Scientific Research NWO, The Netherlands Graduate School for Astronomy NOVA, and SOZOU. We thank Joeri van Leeuwen for an astute comment. The collaboration between Utrecht and Stockholm was part of the European Solar Magnetometry Network supported by the European Commission under contract ERBFMRXCT980190 and is part of the European Solar Magnetism Network supported by the European Commission under contract HPRN-CT-2002-00313.

\section{References}

Alissandrakis, C. E., Georgakilas, A. A., \& Dialetis, D. 1992, Sol. Phys., 138, 93

Athay, R. G. 1970, Sol. Phys., 11, 347

Balthasar, H. 1999, Sol. Phys., 187, 389

Beckers, J. M., \& Tallant, P. E. 1969, Sol. Phys., 7, 351

de Boer, C. R. 1993, Ph.D. Thesis, Göttingen

de Boer, C. R. 1996, A\&AS, 120, 195

de Boer, C. R., \& Kneer, F. 1992, A\&A, 264, L24

Bogdan, T. J. 2000, Sol. Phys., 192, 373

Bogdan, T. J., Rosenthal, C. S., Carlsson, M., et al. 2002, Astron. Nachr., 323, 196

Brisken, W. F., \& Zirin, H. 1997, ApJ, 478, 814

Brynildsen, N., Maltby, P., Fredvik, T., \& Kjeldseth-Moe, O. 2002, Sol. Phys., 207, 259

Brynildsen, N., Maltby, P., Leifsen, T., Kjeldseth-Moe, O., \& Wilhelm, K. 2000, Sol. Phys., 191, 129

Carlsson, M., \& Stein, R. F. 1994, in Chromospheric Dynamics, ed. M. Carlsson, Proc. Miniworkshop Oslo, 47

Carlsson, M., \& Stein, R. F. 1997, ApJ, 481, 500

Christopoulou, E. B., Georgakilas, A. A., \& Koutchmy, S. 1999, in Third Advances in Solar Physics Euroconference: Magnetic Fields and Oscillations, ASP Conf. Ser., 184, 103

Christopoulou, E. B., Georgakilas, A. A., \& Koutchmy, S. 2000, A\&A, 354, 305

Christopoulou, E. B., Georgakilas, A. A., \& Koutchmy, S. 2001, A\&A, 375, 617

Collados, M. 2002, Astron. Nachr., 323, 254

Gelfreikh, G. B., Grechnev, V., Kosugi, T., \& Shibasaki, K. 1999, Sol. Phys., 185, 177

Georgakilas, A. A., Christopoulou, E. B., \& Koutchmy, S. 2000, A\&A, 363, 306

Georgakilas, A. A., Muglach, K., \& Christopoulou, E. B. 2002, ApJ, 576,561

Gurman, J. B., Leibacher, J. W., Shine, R. A., Woodgate, B. E., \& Henze, W. 1982, ApJ, 253, 939

Hammerschlag, R. H., \& Bettonvil, F. C. M. 1998, New Astron. Rev., 42,485

Hofmann, K., \& Weigelt, G. 1986, A\&A, 167, L15

Kneer, F., Mattig, W., \& Uexküll, M. V. 1981, A\&A, 102, 147 
Kupke, R., Labonte, B. J., \& Mickey, D. L. 2000, Sol. Phys., 191, 97 Lites, B. W. 1986, ApJ, 301, 1005

Lites, B. W. 1992, in Sunspots: Theory and Observations, ed. J. H. Thomas, \& N. O. Weiss, NATO ASI Series C 375 (Dordrecht: Kluwer), 261

Lites, B. W. 1994, in Chromospheric Dynamics, ed. M. Carlsson, Proc. Miniworkshop Oslo, 1

Lites, B. W., Rutten, R. J., \& Kalkofen, W. 1993, ApJ, 414, 345

Lites, B. W., Thomas, J. H., Bogdan, T. J., \& Cally, P. S. 1998, ApJ, 497, 464

López Ariste, A., Socas-Navarro, H., \& Molodij, G. 2001, ApJ, 552, 871

von der Lühe, O. 1984, J. Opt. Soc. Am., 1, 510

Maltby, P., Brynildsen, N., Fredvik, T., Kjeldseth-Moe, O., \& Wilhelm, K. 1999, Sol. Phys., 190, 437

Maltby, P., Brynildsen, N., Kjeldseth-Moe, O., \& Wilhelm, K. 2001, A\&A, 373, L1

McIntosh, P. S. 1990, Sol. Phys., 125, 251

McIntosh, S. W., \& Judge, P. G. 2001, ApJ, 561, 420

McIntosh, S. W., Bogdan, T. J., Cally, P. S., et al. 2001, ApJ, 548, $\mathrm{L} 237$

Nindos, A., Alissandrakis, C. E., Gelfreikh, G. B., Bogod, V. M., \& Gontikakis, C. 2002, A\&A, 386, 658

Parker, E. N. 1979, ApJ, 234, 333

Rosenthal, C. S., Bogdan, T. J., Carlsson, M., et al. 2002, ApJ, 564, 508

Rouppe van der Voort, L. H. M. 2002, A\&A, 389, 1020

Ruiz Cobo, B., \& Del Toro Iniesta, J. C. 1992, ApJ, 398, 375

Rutten, R. J. 1996, in Stellar Surface Structure, ed. K. G. Strassmeier, \& J. L. Linsky (Kluwer, Dordrecht), IAU Proc. Symp., 176, 385

Scharmer, G., \& Löfdahl, M. 1991, Adv. Space Res., 11, 129
Scharmer, G. B., Bjelksjö, K., Korhonen, T. K., Lindberg, B., \& Pettersson, B. 2002, in Innovative Telescopes and Instrumentation for Solar Astrophysics, ed. S. Keil, \& S. Avakyan, Proc. SPIE, 4853-47, in press

Scharmer, G. B., Pettersson, L., Brown, D. S., \& Rehn, J. 1985, Appl. Opt., 24, 2558

Settele, A., Sigwarth, M., \& Muglach, K. 2002, A\&A, 392, 1095

Schrijver, C. J., Title, A. M., Berger, T. E., et al. 1999, Sol. Phys., 187, 261

Shand, M., Wei, W., \& Scharmer, G. 1995, in Proc. SPIE, ed. J. Schewel, 2607, 145

Shibasaki, K. 2001, ApJ, 550, 1113

Shine, R., Title, A., Tarbell, T., et al. 1994, ApJ, 430, 413

Socas-Navarro, H., Trujillo Bueno, J., \& Ruiz Cobo, B. 2000a, ApJ, 544,1141

Socas-Navarro, H., Trujillo Bueno, J., \& Ruiz Cobo, B. 2000b, Science, 288, 1396

Socas-Navarro, H., Trujillo Bueno, J., \& Ruiz Cobo, B. 2001, ApJ, 550,1102

Spruit, H. C. 1981, in The Physics of Sunspots, ed. L. E. Cram, \& J. H. Thomas, Sacr. Peak Workshop, 359

Staude, J. 1999, in Third Advances in Solar Physics Euroconference: Magnetic Fields and Oscillations, ASP Conf. Ser., 184, 113

Staude, J. 2002, Astron. Nachr., 323, 317

Sütterlin, P., \& Wiehr, E. 2000, Sol. Phys., 194, 35

Thomas, J. H., Cram, L. E., \& Nye, A. H. 1984, ApJ, 285, 368

Thomas, J. H., \& Weiss, N. O. 1992, Sunspots: Theory and Observations (Kluwer, Dordrecht)

Tziotziou, K., Tsiropoula, G., \& Mein, P. 2002, A\&A, 381, 279

Weigelt, G. P. 1977, Opt. Comm., 21, 55

Zhugzhda, Y. D., Balthasar, H., \& Staude, J. 2000, A\&A, 355, 347 